\title{
Recurrent Seizure: An Uncommon Presentation of Post Thyroidectomy Hypoparathyroidism
}

\author{
ABDUL WADUD CHOWDHURY, ${ }^{1}$ SABBI HA NADIA MAJ UMDER, ${ }^{2}$ MD GAFFAR AMI N, ${ }^{3} \mathrm{KAZ}$ NAZRULISLAM, ${ }^{3}$ \\ MOHAMMED ABAYE DEEN SALEH, ${ }^{4}$ KHANDKER MD NURUS SABAH, ${ }^{5}$ MD NAZ MUDDI N ${ }^{6}$
}

\begin{abstract}
:
Basal ganglia calcification is associated with chronic hypoparathyroidism. We report a case of 37 year old lady with long standing iatrogenic hypoparathyroidism following total thyroidectomy. The clinical evaluation revealed neurological symptoms but without any neurological deficit. The CT scan of head showed calcification in caudate nucleus and part of lentiform nucleus of basal ganglia and in the cortical and subcortical white matter. Detection of brain calcinosis in patient's who had total thyroidectomy can guide clinicians to further investigation for possible hypoparathyroidism.
\end{abstract}

Key words: Basal ganglia calcification, brain calcinosis, iatrogenic hypoparathyroidism.

\section{Introduction:}

Physiological intracranial calcification is rare (.3-1.5\%). ${ }^{1}$ Pathological basal ganglia calcification occurs due to various causes such as metabolic disorders, infectious diseases and genetic diseases. Intracranial calcification is a rare manifestation of post operative hypoparathyroidism. Patients with basal ganglia calcification can present with myriad, atypical symptoms or can remain asymptomatic till detected by incidental investigations. ${ }^{1}$ Here we are reporting a young woman presenting with persistent circumoral and fingertip paraesthesia and occasional tetanic seizure due to post thyroidectomy hypoparathyroidism induced hypocalcaemia and basal ganglia calcification.

\section{Case report:}

A 37 year old housewife presented to the outpatient clinic of Dhaka Medical College Hospital (DMCH) with the complaints of fever with rash for 10 days and a brief episode of loss of consciousness. She was admitted to medicine

1. Professor, Dept. of Cardiology, Dhaka Medical College and Hospital

2. Honorary Medical Officer, Dept. of Cardiology, Dhaka Medical College and Hospital

3. Junior consultant, Dept. of Cardiology, Dhaka Medical College and Hospital

4. Registrar, Dept. of Cardiology, Dhaka Medical College and Hospital

5. Asst. Prof, Dept. of Cardiology, Dhaka Medical College and Hospital

6. Indoor Medical Officer, Dept. of Cardiology, Dhaka Medical College and Hospital

Address of Correspondence: Dr Sabbiha Nadia Majumder, Honorary Medical Officer, Dept. of Cardiology, Dhaka Medical College and Hospital. Cell: 01682076378, e-mail: snadiamajumder @gmail.com. department and was diagnosed as a case of varicella and treated accordingly. During her stay, she had two episodes of generalized tonic clonic seizure and tongue bite and was managed symptomatically. Three days after admission, during her convalescent period, she experienced ischaemic type chest pain without significant ECG changes. Her troponin I was slightly raised to $0.71 \mathrm{ng} / \mathrm{dl}$ (cut off $>0.60 \mathrm{ng} / \mathrm{dl}$ ). She was transferred to Cardiology department of $\mathrm{DMCH}$ as a case of non ST segment elevated myocardial infarction (NSTEMI).

She gave a history of total thyroidectomy in 2008 and treated with levothyroxine replacement therapy. Her clinical course during or after surgery was unknown as she did not have any proper post operative follow-up and could not show any proper documentation. Nevertheless she reported a rapidly developing large goiter with mechanical discomfort with difficulty in swallowing and respiration before surgery but without history of exophthalmos or weight loss. She was prescribed calcium supplementation after surgery which she stopped herself after a month. So according to patient's history, multinodular goiter with mechanical compression is presumed to be the indication for total thyroidectomy. The patient complained of fatigability as well as tingling and numbness in the fingertips and around the mouth following surgery. She also had occasional tetanic seizures (2-3 times a year) since then. During every attack, she was admitted to the nearby hospital and treated with I/V diazepam and calcium gluconate. According to her family members, she had no feature of dementia and mental incapability in her everyday life.

On examination, there was no dismorphic feature. Trousseau's sign and Chvostek's signs were positive. 


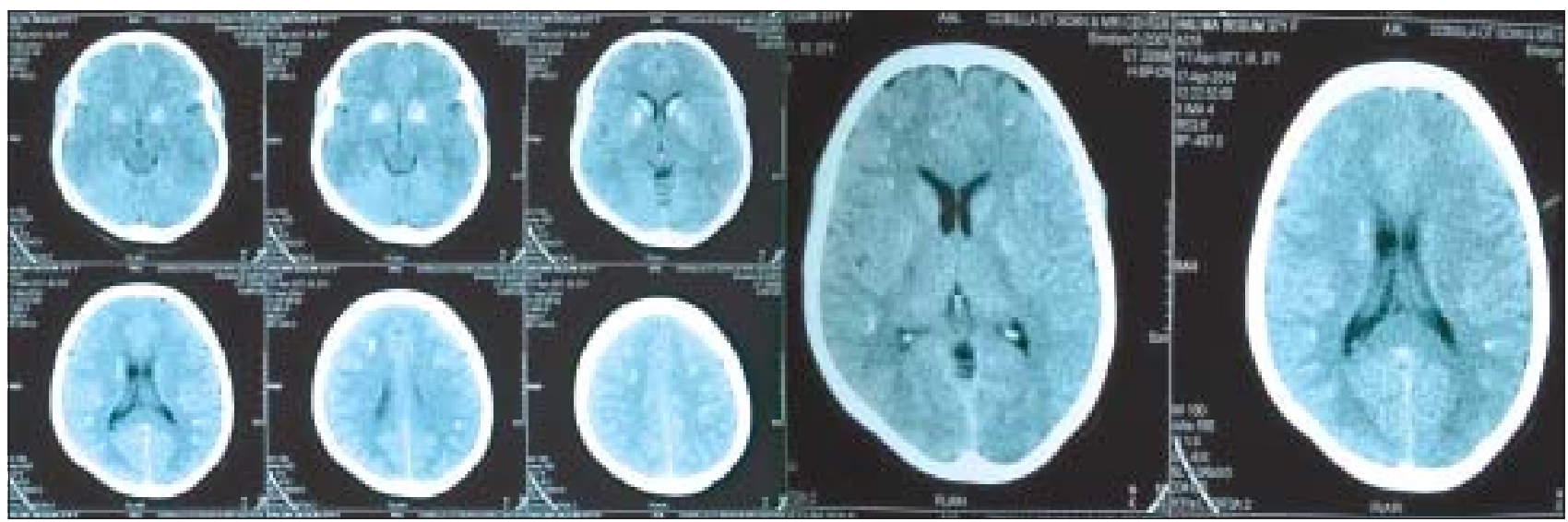

Fig.-1: CT scan of head showing bilateral basal ganglia calcification and scattered bilateral cortical and subcortical white matter calcification in parietal and frontal lobe.

Neurological examination was normal with no extrapyramidal signs. Fundoscopy was normal. Cardiovascular system examination revealed normal findings (blood pressure- 120/ $80 \mathrm{mmHg}$, pulse- 80 beats/minute)

On investigation, her corrected calcium level was $3.89 \mathrm{mg} / \mathrm{dl}$ (8.1-10.4 mg/dl), serum albumin: $36 \mathrm{~g} / \mathrm{l}(35-50 \mathrm{~g} / \mathrm{l})$, serum magnesium: $1.60 \mathrm{mg} / \mathrm{dl}(1.6-2.3 \mathrm{mg} / \mathrm{dl})$, serum phosphate: 7.21 $\mathrm{mg} / \mathrm{dl}$ (2.5-4.65 mg/dl), serum parathyroid hormone: $<3 \mathrm{pg} / \mathrm{ml}$ (11-67 pg/ml), serum TSH: $2.55 \mathrm{IU} / \mathrm{ml}$, FT3: $0.48 \mathrm{nmol} / \mathrm{l}$, FT4: $103.60 \mathrm{nmol} /$, lipid profile: Cholesterol - $277 \mathrm{mg} / \mathrm{dl}$ (120-200 $\mathrm{mg} / \mathrm{dl}$ ); HDL-Cholesterol- $46 \mathrm{mg} / \mathrm{dl}$ (>40mg/dl); LDLCholesterol-192mg/dl (<130mg/dl); Triglycerides-195 mg/dl (fasting: 10-190 mg/dl), fasting plasma glucose: $4 \mathrm{mmol} / \mathrm{l}$, two hours after 75 gm glucose: $5.2 \mathrm{mmol} / \mathrm{l}$, HbA1c: $5.4 \%$ ( $<6$ $\%$ ), serum creatinine: $0.8 \mathrm{mg} / \mathrm{dl}(0.4-1.2 \mathrm{mg} / \mathrm{dl})$. The rest of the lab evaluation was unremarkable.

CT scan of head revealed bilateral basal ganglia calcification and scattered bilateral cortical and subcortical white matter calcification in parietal and frontal lobe (Figure 1). Her repeat troponin I was normal. Her echocardiographic report showed mildly hypokinetic anteroseptal wall with mild LV systolic dysfunction (EF 48\%).

The clinical presentation of our patient was attributed to bilateral basal ganglia calcification due to hypocalcaemia with hyperphosphataemia resulting from thyroid surgery.

She was given cardiac medications (metoprolol, dual antiplatelets, low molecular weight heparin and statin) as well as intravenous calcium gluconate followed by oral calcium supplementation (3g/day). This normalized her serum calcium level $(8.9 \mathrm{mg} / \mathrm{dl})$ with complete recovery of her paraesthesia (both perioral and fingertips). To prevent further complication from hypoparathyroidism, the patient was prescribed calcitriol 0.8 microgram/day and calcium $1 \mathrm{gm} /$ day. The patient was followed up after 15 days and she remained in good mental and physical health.

\section{Discussion:}

Hypoparathyroidism is an endocrine disorder caused by autoimmune, congenital (Digeorge's syndrome), iatrogenic causes (drugs, removal of parathyroid glands during thyroid or parathyroid surgery, radiation), infiltration of parathyroid glands (metastatic carcinoma, Wilson's disease, sarcoidosis), suppression of parathyroid function such as in hypomagnesemia, HIV/AIDS or idiopathic mechanism, pseudohypoparathyroidism (failure of target cell response to parathormone) and pseudopseudohypoparathyroidism. ${ }^{2,3,4}$

Radiologically, hypoparathyroidism of any type causes calcification most often in the basal ganglia, the most common site is often globus pallidus. It can also occur in cerebellum, subcortical white matter, corona radiata and thalamus. ${ }^{2,3,4}$

Causes of symmetrical calcaification of basal ganglia include: physiological, probably related to aging, hypoparathyroidism, hyperparathyroidism, familial idiopathic cerebral calcification (Fahr's syndrome), birth anoxia, carbon monoxide poisoning/intoxication, lead poisoning, tuberous sclerosis, Cockayne's syndrome, post infectious, acquired immune deficiency syndrome (specially in children), radiation therapy, methotrexate therapy, Kearn Sayre syndrome and other mitochondrial diseases, familial encephalopathies and down's syndrome.

In skeletal survey, calavarial thickening, soft tissue calcification and premature closure of the epiphysis may or may not be present in all type of hypoparathyroidism. Patients commonly have mild or diffuse osteosclerosis too. 
Pseudohypoparathyroidism and pseudopseudohypoparathyroidism classically have metacarpal/ metatarsal shortening and exostosis formation. Most commonly involved are the first and fourth metacarpal and metatarsal bone. ${ }^{1,5,6}$

Postoperative hypoparathyroidism is the most common complication of complete or near complete extirpation of thyroid gland by destruction or vascular compromise of parathyroid tissue. Several thyroid condition such as Grave's disease, thyrotoxicosis as a result of hyperactive thyroid adenoma, recurrent goiter, thyroid carcinoma carry a higher risk to develop transient and permanent hypoparathyroidism post operatively.

The main clinical feature of hypoparathyroidism is a result of induced hypocalcaemia and can range from a life threatening condition to an asymptomatic lab finding. Hypocalcaemia most commonly presents with paraesthesia, cramps, muscle spasms, circumoral numbness and seizure. But patients can also present with laryngospasm, neuromuscular irritability, cognitive impairment, personality disturbances, prolonged QT interval or electrocardiographic changes that mimic myocardial infarcton and heart failure. ${ }^{2,4,5}$

Intracranial calcification is one of the features of hypocalcaemia and the calcifications typically involve the basal ganglia, thalami and cerebellum. In our patient, calcinosis exceeds the common brain location and involves the subcortical white matters of frontal and parietal lobes. A review of literature reveals only a few case reports of excessive calcification of subcortical white matter in post operative hypoparathyroidism.

The most commonly reported manifestations of post operative hypoparathyroidism with basal ganglia calcification are parkinsonism and seizures. There are also reports of cognitive impairment and even intracerebral haemorrhage. The remarkable point of our case is the discordance between extensive imaging findings and paucity of clinical symptoms and signs. Despite the brain calcification, the patient had only circumoral and fingertip paresthesia and occasional seizure without any persistent neurological deficit. The patient developed chest pain due to NSTEMI in early ages. She had dyslipidemia and also hyperphosphatemia.

The pathogenic mechanism of brain calcinosis in post operative hypoparathyroidism is not yet defined. Microscopic colloid deposition around cerebral blood vessels is followed by calcification most commonly in the basal ganglia. The progression of basal ganglia calcification is related to calcium phosphate ratio. Thus a strict control of hypocalcaemia and hypophosphatemia upon diagnosis is mandatory. ${ }^{7,8,9}$

\section{Conclusion:}

Chronic hypocalcaemia due to postoperative hypoparathyroidism can remain subclinical for long. Since adequate treatment of hypoparathyroidism may lead to clinical improvement and due to its rarity, detection of intracranial calcification can be the trigger for further investigation with measurement of calcium and phosphorus level and parathyroid hormone. Proper calcium supplementation can easily protect the patient from further complication of chronic hypoparathyroidism. ${ }^{10}$

\section{Conflict of interest: None.}

\section{References:}

1. Jorens PG, Appel BJ, Hilte FA, Mahler C, De Deyn PP. Basal ganglia calcifications in postoperative hypoparathyroidism: a case with unusual characteristics. 1991; 83(2): 137-40.

2. Kazis AD. Contribution of CT scan to the diagnosis of Fahr's syndrome. Acta Neurol Scand 1985; 71(3): 206-211.

3. Fénelon G, Gray F, Paillard F, Thibierge M, Mahieux F, Guillani A. A prospective study of patients with CT detected pallidal calcifications. J Neurol Neurosurg Psychiatry 1993; 56(6): 622-625.

4. Murphy MJ. Clinical correlations of CT scan-detected calcifications of the basal ganglia. Ann Neurol 1979; 6(6): 507-511.

5. Vakaet A, Rubens R, de Reuck J, vander Eecken H. Intracranial bilateral symmetrical calcification on CTscanning. A case report and a review of the literature. Clin Neurol Neurosurg 1985; 87 (2): 103-11.

6. Litvin Y, Rosler A, Bloom RA. Extensive cerebral calcification in hypoparathyroidism. Neuroradiology 1981; 21 (5): 271-2.

7. Lang C, Huk W, Pichl J. Comparison of extensive brain calcification in postoperative hypoparathyrodism on CT and NMR scan. Neuroradiology 1989; 31(1): 29-32.

8. Litvin Y, Rosler A, Bloom RA. Extensive cerebral calcification in hypoparathyroidism. Neuroradiology. 1981; 21(5): 271-272.

9. Reddy ST, Merrick RD. Hypoparathyroidism, intracranial calcification, and seizures 61 years after thyroid surgery. Tennessee Medicine 1999; 92(9): 341-342.

10. Hypoparathyroidism, pseudohypoparathyroidism, pseudopseudo hypoparathyroidism, chapter metabolic and endocrine disease of the skeleton.by J.B.Vogler III and J.H.Kim -Diagnostic Radiology 4th edition volume three. 